\title{
Mixing lossy and perfect fifo channels ${ }^{\star}$
}

\author{
P. Chambart and Ph. Schnoebelen \\ LSV, ENS Cachan, CNRS \\ 61, av. Pdt. Wilson, F-94230 Cachan, France \\ email: \{chambart|phs\} @1sv.ens-cachan.fr
}

\begin{abstract}
We consider asynchronous networks of finite-state systems communicating via a combination of reliable and lossy fifo channels. Depending on the topology, the reachability problem for such networks may be decidable. We provide a complete classification of network topologies according to whether they lead to a decidable reachability problem. Furthermore, this classification can be decided in polynomial-time.
\end{abstract}

\section{Introduction}

Fifo channels. Channel systems, aka "communicating finite-state machines", are a classical model for protocols where components communicate asynchronously via fifo channels [BZ83]. When the fifo channels are unbounded, the model is Turing-powerful since channels can easily be used to simulate the tape of a Turing machine.

It came as quite a surprise when Abdulla and Jonsson [AJ96,ACBJ04], and independently Finkel et al. [Fin94], showed that lossy channel systems (LCS's), i.e., channel systems where one assumes that the channels are unreliable so that messages can be lost nondeterministically, are amenable to algorithmic verification (see also [Pac87]). The model has since been extended in several directions: message losses obeying probability laws [Sch04,ABPJ05,ABRS05,BBS07], channels with other kinds of unreliability $\left[\mathrm{CFP} 96, \mathrm{BMO}^{+} 08\right]$, etc.

How this unreliability leads to decidability is paradoxical, and hard to explain in high-level, non-technical terms. It certainly does not make the model trivial: we recently proved that LCS verification is exactly at level $\mathcal{F}_{\omega^{\omega}}$ in the Extended Grzegorczyk Hierarchy, hence it is not primitive-recursive, or even multiply-recursive [CS08b].

An ubiquitous model. In recent years, lossy channels have shown up in unexpected places. They have been used in reductions showing hardness (or less frequently decidability) for apparently unrelated problems in modal logics [Kur06], in temporal logics [OW07], in timed automata [LW08], in data-extended models [JL07], etc. More and more, LCS's appear to be a pivotal model whose range goes far beyond asynchronous protocols.

Fueling this line of investigation, we recently discovered that the "Regular Post Embedding Problem", a new decidable variant of Post's Correspondence Problem, is equivalent (in a non-trivial way) to LCS reachability [CS07,CS08a]. This discovery

\footnotetext{
* Work supported by the Agence Nationale de la Recherche, grant ANR-06-SETIN-001.
} 
was an unexpected outcome of our study of unidirectional channel systems (UCS), where a Sender can send messages to a Receiver via two fifo channels, one reliable and one lossy, but where there is no communication in the other direction (see $T_{2}^{d}$ in Fig. 1 below). As far as we know, this simple arrangement had never been studied before.

Our contribution. This paper considers the general case of mixed channel systems, where some channels are reliable and some are lossy. These systems can be Turingpowerful (one process using one reliable fifo buffer is enough) but not all network topologies allow this (e.g., systems with only lossy channels, or systems where communication is arranged in a tree pattern with no feedback, or UCS's as above). We provide a complete classification of network topologies according to whether they lead to undecidable reachability problems, or not. This relies on original and non-trivial transformation techniques for reducing large topologies to smaller ones while preserving decidability.

This is a fundamental study, aiming at understanding the fifo channel model in presence of message losses. A long-term goal would be to converge towards a uniform treatment and understanding of the various decidable families of channel systems, including half-duplex systems [CF05], linear and monogeneous systems [JJ93], etc.

Beyond providing a complete classification, the present contribution has several interesting outcomes. First, we discovered new decidable arrangements of channel systems, as well as new undecidable ones, and these new results are often surprising. They enlarge the existing toolkit currently used when transferring results from channel systems to other areas, according to the "ubiquitous model" slogan. Secondly, the transformation techniques we develop may eventually prove useful for reducing/delaying the combinatorial explosion one faces when verifying asynchronous protocols.

Outline of the paper. We describe mixed channel systems and their topologies in Section 2 and provide in Section 3 a few original results classifying the basic topologies to which we reduce larger networks. Section 4 shows that "fusing essential channels" preserves decidability. An additional "splitting" technique is described in Section 5. After these three sections, we have enough technical tools at hand to describe our main result, the complete classification method, and prove its correctness in Sections 6 and 7. Proofs omitted in the main text are given in the technical appendix.

\section{Systems with reliable and lossy channels}

We classify channel systems according to their network topology, which is a graph describing who are the participant processes and what channels they are connected to.

\subsection{Network topologies}

Formally, a network topology, or shortly a topology, is a tuple $T=\langle N, R, L, s, d\rangle$ where $N, R$ and $L$ are three mutually disjoint finite sets of, respectively, nodes, reliable channels, and lossy channels, and where, writing $C \stackrel{\text { def }}{=} R \cup L$ for the set of channels, $s, d$ : 
$C \rightarrow N$ are two mappings that associate a source and a destination node to each channel. We do not distinguish between isomorphic topologies since $N, R$ and $L$ simply contain "names" for nodes and channels: these are irrelevant here and only the directed graph structure with two types of edges matters.

Graphical examples of simple topologies will be found below: we use dashed arrows to single out the lossy channels (reliable channels are depicted with full arrows).

\subsection{Mixed channel systems and their operational semantics}

Assume $T=\langle N, R, L, s, d\rangle$ is a topology with $n$ nodes, i.e., with $N=\left\{P_{1}, P_{2}, \ldots, P_{n}\right\}$. Write $C=R \cup L$ for the set of channels. A mixed channel system (MCS) having topology $T$ is a tuple $S=\left\langle T, M, Q_{1}, \Delta_{1}, \ldots, Q_{n}, \Delta_{n}\right\rangle$ where $M=\{\mathrm{a}, \mathrm{b}, \ldots\}$ is a finite message alphabet and where, for $i=1, \ldots, n, Q_{i}$ is the finite set of (control) states of a process (also denoted $P_{i}$ ) that will be located at node $P_{i} \in N$, and $\Delta_{i}$ is the finite set of transition rules, or shortly "rules", governing the behaviour of $P_{i}$. A rule $\delta \in \Delta_{i}$ is either a writing rule of the form $\left(q, c, !\right.$, a,$\left.q^{\prime}\right)$, usually denoted " $q \stackrel{c ! \mathrm{a}}{\rightarrow} q^{\prime}$ ", with $q, q^{\prime} \in Q_{i}, s(c)=P_{i}$ and a $\in M$, or it is a reading rule $\left(q, c, ?, \mathrm{a}, q^{\prime}\right)$, usually denoted " $q \stackrel{c ? \mathrm{a}}{\longrightarrow} q^{\prime \prime}$, , with this time $d(c)=P_{i}$. Hence the way a topology $T$ is respected by a channel system is via restrictions upon the set of channels to which a given participant may read from, or write to.

Our terminology "mixed channel system" is meant to emphasize the fact that we allow systems where lossy channels coexist with reliable channels.

The behaviour of some $S=\left\langle T, M, Q_{1}, \Delta_{1}, \ldots, Q_{n}, \Delta_{n}\right\rangle$ is given under the form of a transition system. Assume $C=\left\{c_{1}, \ldots, c_{k}\right\}$ contains $k$ channels. A configuration of $S$ is a tuple $\sigma=\left\langle q_{1}, \ldots, q_{n}, u_{i}, \ldots, u_{k}\right\rangle$ where, for $i=1, \ldots, n, q_{i} \in Q_{i}$ is the current state of $P_{i}$, and where, for $i=1, \ldots, k, u_{i} \in M^{*}$ is the current contents of channel $c_{i}$.

Assume $\sigma=\left\langle q_{1}, \ldots, q_{n}, u_{i}, \ldots, u_{k}\right\rangle$ and $\sigma^{\prime}=\left\langle q_{1}^{\prime}, \ldots, q_{n}^{\prime}, u_{i}^{\prime}, \ldots, u_{k}^{\prime}\right\rangle$ are two configurations of some system $S$ as above, and $\delta \in \Delta_{i}$ is a rule of participant $P_{i}$. Then $\delta$ witnesses a transition between $\sigma$ and $\sigma^{\prime}$, also called a step, and denoted $\sigma \stackrel{\delta}{\rightarrow} \sigma^{\prime}$, if and only if

- the control states agree with, and are modified according to $\delta$, i.e., $q_{i}=q, q_{i}^{\prime}=q^{\prime}$, $q_{j}=q_{j}^{\prime}$ for all $j \neq i$

- the channel contents agree with, and are modified according to $\delta$, i.e., either

- $\delta=\left(q, c_{l}, ?, \mathrm{a}, q^{\prime}\right)$ is a reading rule, and $u_{l}=\mathrm{a} \cdot u_{l}^{\prime}$, or

- $\delta=\left(q, c_{l}, !\right.$, a,$\left.q^{\prime}\right)$ is a writing rule, and $u_{l}^{\prime}=u_{l}$.a, or $c_{l} \in L$ is a lossy channel and $u_{l}^{\prime}=u_{l}$;

in both cases, the other channels are untouched: $u_{j}^{\prime}=u_{j}$ for all $j \neq l$.

Such a step is called " $a$ step by $P_{i}$ " and we say that its effect is "reading a on $c$ ", or "writing a to $c$ ", or "losing a". A run (from $\sigma_{0}$ to $\sigma_{p}$ ) is a sequence of steps of the form $r=\sigma_{0} \stackrel{\delta_{1}}{\rightarrow} \sigma_{1} \stackrel{\delta_{2}}{\rightarrow} \sigma_{2} \cdots \stackrel{\delta_{p}}{\rightarrow} \sigma_{p}$, sometimes shortly written $\sigma_{0} \stackrel{*}{\rightarrow} \sigma_{p}$. A run is perfect if none of its steps loses a message.

Remark 2.1. With this operational semantics for lossy channels, messages can only be lost when a rule writes them to a channel. Once inside the channels, messages can 
only be removed by reading rules. This definition is called the write-lossy semantics for lossy channels: it differs from the more classical definition where messages in lossy channels can be lost at any time. We use it because it is the most convenient one for our current concerns, and because this choice does not impact the reachability questions we consider (see [CS08b, Appendix A] for a formal comparison).

\subsection{The reachability problem for network topologies}

The reachability problem for mixed channel systems asks, for a given $S$ and two configurations $\sigma_{\text {init }}=\left\langle q_{1}, \ldots, q_{n}, \varepsilon, \ldots, \varepsilon\right\rangle$ and $\sigma_{\text {final }}=\left\langle q_{1}^{\prime}, \ldots, q_{n}^{\prime}, \varepsilon, \ldots, \varepsilon\right\rangle$ in which the channels are empty, whether $S$ has a run from $\sigma_{\text {init }}$ to $\sigma_{\text {final }}$. That we restrict reachability questions to configurations with empty channels ( $\varepsilon$ denotes the empty word in $M^{*}$ ) is technically convenient, but it is no real loss of generality.

The reachability problem for a topology $T$ is the restriction of the reachability problem to mixed systems having topology $T$. Hence if reachability is decidable for $T$, it is decidable for all MCS's having topology $T$. If reachability is not decidable for $T$, it may be decidable or not for MCS's having topology $T$ (but it must be undecidable for one of them). Clearly, if $T^{\prime}$ is a subgraph of $T$ and reachability is decidable for $T$, then it is for $T^{\prime}$ too.

Our goal is to determine for which topologies reachability is decidable. Let us illustrate the question and outline some of our results. $T_{1}^{\text {ring }}$ is a topology describing a directed ring of processes, where each participant sends to its right-hand neighbour, and receives from its left-hand neighbour. A folk claim is that such cyclic networks have decidable reachability as soon as one channel is lossy (as here with $c_{2}$ ). The proof ideas behind this claim have not been formally published and they do not easily adapt to related questions like "what about $T_{2}^{\text {ring }}$ ?", where a lossy channel in the other direction is

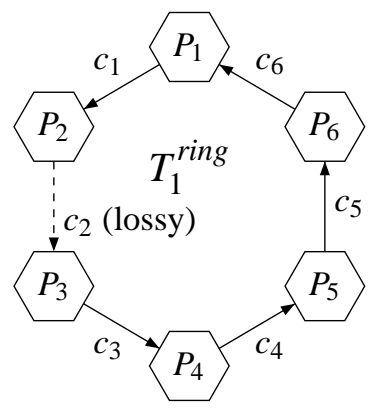
added, or about $T_{3}^{\text {ring }}$ where more channels are lossy in the ring.
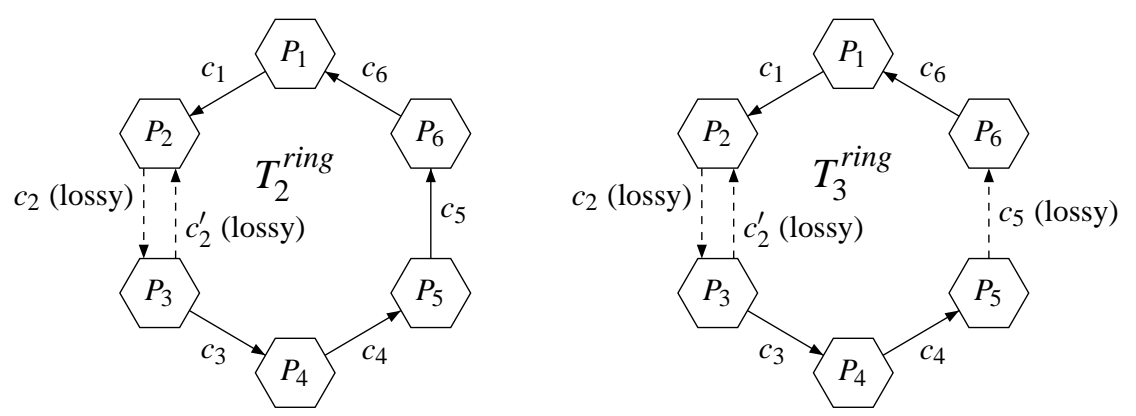

Our techniques answer all three questions uniformly. One of our results states that all channels along the path $c_{3}$ to $c_{4}$ to $c_{5}$ to $c_{6}$ to $c_{1}$ can be fused into a single channel 
going from $P_{3}$ to $P_{2}$ without affecting the decidability of reachability. The transformations are modular (we fuse one channel at a time). Depending on the starting topology, we end up with different two-node topologies, from which we deduce that $T_{1}^{\text {ring }}$ and $T_{3}^{\text {ring }}$ have decidable reachability, while $T_{2}^{\text {ring }}$ does not (see Corollary 4.6 below).

\section{Reachability for basic topologies}

This section is concerned with the basic topologies to which we will later reduce all larger cases.

Theorem 3.1 (Basic topologies). Reachability is decidable for the network topologies $T_{1}^{d}$ and $T_{2}^{d}$ (see Fig. 1). It is not decidable for the topologies $T_{1}^{u}, T_{2}^{u}, T_{3}^{u}, T_{4}^{u}, T_{5}^{u}$, and $T_{6}^{u}$ (see Fig. 2).

We start with the decidable cases:

That $T_{1}^{d}$, and more generally all topologies with only lossy channels (aka LCS's), leads to decidable problems is the classic result from [AJ96].

Regarding $T_{2}^{d}$, we recently proved it has decidable reachability in [CS07], where $T_{2}^{d-}$ systems are called "unidirectional channel systems", or UCS's. Our reason for investigating

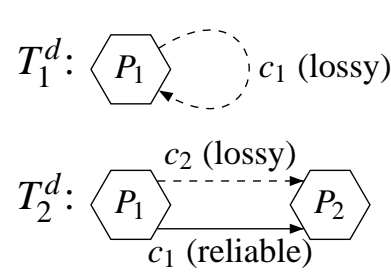

Fig. 1. Basic decidable topologies UCS's was indeed that this appeared as a necessary preparation for the classification of mixed topologies. Showing that $T_{2}^{d}$ has decidable reachability is quite involved, going through the introduction of the "Regular Post Embedding Problem". In addition, [CS07,CS08a] exhibit non-trivial reductions between reachability for UCS's and reachability for LCS's: the two problems are equivalent.

Now to the undecidable cases:

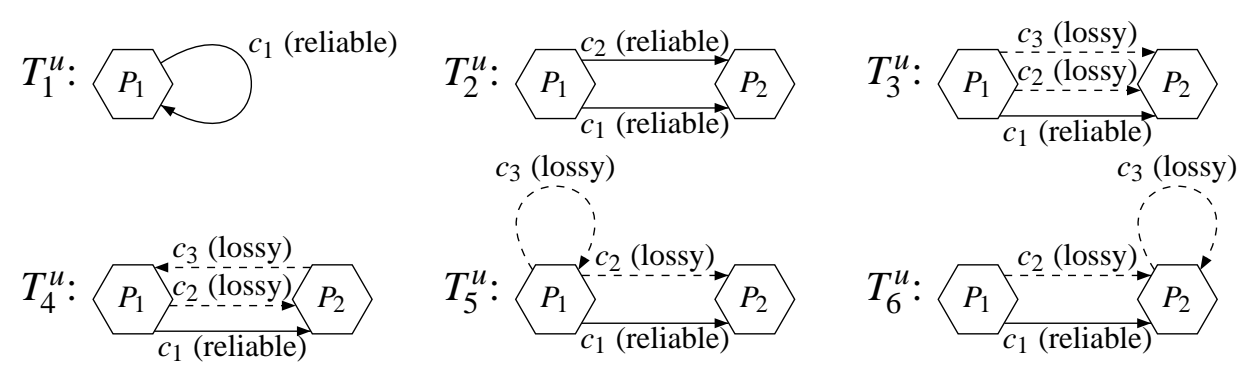

Fig. 2. Basic topologies with undecidable reachability

It is well-known that $T_{1}^{u}$ may lead to undecidable problems [BZ83], and this is also known, though less well, for $T_{2}^{u}$ (restated, e.g., as the non-emptiness problem for the intersection of two rational transductions). The other four results mix lossy and 
reliable channels and are new. We actually prove all six cases in a uniform framework, by reduction from Post's Correspondence Problem, aka PCP, or its directed variant, $P C P_{\text {dir }}$.

Recall that an instance of PCP is a family $x_{1}, y_{1}, x_{2}, y_{2}, \ldots, x_{n}, y_{n}$ of $2 n$ words over some alphabet. The question is whether there is a non-empty sequence (a solution) $i_{1}, \ldots, i_{k}$ of indexes such that $x_{i_{1}} x_{i_{2}} \ldots x_{i_{k}}=y_{i_{1}} y_{i_{2}} \ldots y_{i_{k}} . \mathrm{PCP}_{\text {dir }}$ asks whether there is a directed solution $i_{1}, \ldots, i_{k}$, i.e., a solution such that, in addition, $y_{i_{1}} y_{i_{2}} \ldots y_{i_{h}}$ is a prefix of $x_{i_{1}} x_{i_{2}} \ldots x_{i_{h}}$ for all $h=1, \ldots, k$. It is well-known that $\mathrm{PCP}$ and $\mathrm{PCP}_{\text {dir }}$ are undecidable, and more precisely $\Sigma_{0}^{1}$-complete.

Reducing PCP to $T_{2}^{u}$-networks. With a PCP instance $\left(x_{i}, y_{i}\right)_{i=1, \ldots, n}$, we associate a process $P_{1}$ having a single state $p_{1}$ and $n$ loops $^{1} p_{1} \stackrel{{ }_{1} ! x_{i} c_{2} ! y_{i}}{\longrightarrow} p_{1}$, one for each index $i=$ $1, \ldots, n$. Process $P_{1}$ guesses a solution $i_{1} i_{2} i_{3} \ldots$ and sends the concatenations $x_{i_{1}} x_{i_{2}} x_{i_{3}} \ldots$ and $y_{i_{1}} y_{i_{2}} y_{i_{3}} \ldots$ on, respectively, $c_{1}$ and $c_{2}$. Process $P_{2}$ checks that the two channels $c_{1}$ and $c_{2}$ have the same contents, using reading loops $p_{2} \stackrel{c_{1} ? a c_{2} ? a}{\longrightarrow} p_{2}$, one for each symbol $\mathrm{a}, \mathrm{b}, \ldots$ in the alphabet. An extra control state, for example $p_{1}^{\prime}$ with rules $p_{1}^{\prime} \stackrel{c_{1} ! x_{i} c_{2} ! y_{i}}{\longrightarrow} p_{1}$, is required to check that $P_{1}$ picks a non-empty solution. Then, in the resulting $T_{2}^{u}$ network, $\left\langle p_{1}^{\prime}, p_{2}, \varepsilon, \varepsilon\right\rangle \stackrel{*}{\rightarrow}\left\langle p_{1}, p_{2}, \varepsilon, \varepsilon\right\rangle$ if and only if the PCP instance has a solution.

Reducing PCP to $T_{3}^{u}$-networks. For $T_{3}^{u}$, the same idea is adapted to a situation with three channels, two of which are lossy. Here $P_{1}$ has rules $p_{1} \stackrel{c_{2} ! x_{i} c_{3} ! y_{i} c_{1} ! 1^{\left|x_{i} y_{i}\right|}}{\longrightarrow} p_{1}$. Thus $P_{1}$ sends $x_{i}$ and $y_{i}$ on lossy channels and simultaneously sends the number of letters in unary ( 1 is a special tally symbol) on $c_{1}$, the perfect channel. $P_{2}$ matches these with reading loops of the form $p_{2} \stackrel{c_{1} ? 11 c_{2} \text { ?a } c_{3} \text { ?a }}{\longrightarrow} p_{2}$ for each letter a. If $P_{2}$ can consume all 1 's out of $c_{1}$, this means that no message has been lost on the lossy channels, and then $P_{2}$ really witnessed a solution the PCP instance.

Reducing $\mathrm{PCP}_{\text {dir }}$ to $T_{1}^{u}$-networks. For $T_{1}^{u}$, we consider the directed $\mathrm{PCP}_{\text {dir }} . P_{1}$ has $n$ loops $p_{1} \stackrel{c_{1} ! x_{i} c_{1} ? y_{i}}{\longrightarrow} p_{1}$ where the guessing and the matching is done by a single process. Since at any step $h=1, \ldots, k$ the concatenation $x_{i_{1}} x_{i_{2}} \ldots x_{i_{h}}$ is (partly) consumed while matching for $y_{i_{1}} y_{i_{2}} \ldots y_{i_{h}}$, only directed solutions will be accepted.

Reducing $\mathrm{PCP}_{\text {dir }}$ to $T_{5}^{u}$-networks. For $T_{5}^{u}$ too, we start from $\mathrm{PCP}_{\text {dir }}$ and use a variant of the previous counting mechanism to detect whether some messages have been lost. $P_{1}$ has rules of the form $p_{1} \stackrel{c_{3} ! 1^{\left|x_{i}\right|} c_{1} ! x_{i} c_{3} ? 1^{\left|y_{i}\right|} c_{2} ! y_{i}}{\longrightarrow} p_{1}$, i.e., it sends $x_{i}$ on $c_{1}$ (the reliable channel) and $y_{i}$ on $c_{2}$ (unreliable) while $P_{2}$ checks the match with loops $p_{2} \stackrel{c_{1} \text { ?a } c_{2} \text { ?a }}{\longrightarrow} p_{2}$. In addition, $P_{1}$ also maintains in $c_{3}$ a count of the number of symbols written to $c_{1}$ minus

\footnotetext{
${ }^{1}$ Transition rules like " $p_{1} \stackrel{c_{1} ! x_{i} c_{2} ! y_{i}}{\longrightarrow} p_{1}$ " above, where several reads and writes are combined in a same rule, and where one writes or reads words rather than just one message at a time, are standard short-hand notations for sequences of rules using intermediary states that are left implicit. We avoid using this notation in situations where the specific ordering of the combined actions is important as, e.g., in $(*)$ below.
} 
the number of symbols written to $c_{2}$, or $\#_{h} \stackrel{\text { def }}{=}\left|x_{i_{1}} \ldots x_{i_{h}}\right|-\left|y_{i_{1}} \ldots y_{i_{h}}\right|$. The counting scheme forbids partial sequences $y_{i_{1}} \ldots y_{i_{h}}$ that would be longer than the corresponding $x_{i_{1}} \ldots x_{i_{h}}$, but this is right since we look for directed solutions. If tally symbols on $c_{3}$ are lost, or if part of the $y_{i}$ 's on $c_{2}$ are lost, then it will never be possible for $P_{2}$ to consume all messages from $c_{1}$. Finally a run from $\left\langle p_{1}^{\prime}, p_{2}, \varepsilon, \varepsilon, \varepsilon\right\rangle$ to $\left\langle p_{1}, p_{2}, \varepsilon, \varepsilon, \varepsilon\right\rangle$ must be perfect and witness a directed solution.

Reducing $\mathrm{PCP}_{\text {dir }}$ to $T_{6}^{u}$-networks. For $T_{6}^{u}$, we adapt the same idea, this time having $P_{2}$ monitoring the count $\#_{h}$ on $c_{3}$. $P_{1}$ has loops $p_{1} \stackrel{c_{1} ! x_{i} 1^{\left|y_{i}\right|} c_{2} ! y_{i} 1^{\left|x_{i}\right|}}{\longrightarrow} p_{1}$ where a guessed solution is sent on $c_{1}$ and $c_{2}$ with interspersed tally symbols. The guessed solution is checked with the usual loops $p_{2} \stackrel{c_{1} \text { ?a } c_{2} \text { ?a }}{\longrightarrow} p_{2}$. The 1 's on $c_{2}$ are stored to $c_{3}$ and matched (later) with the 1 's on $c_{1}$ via two loops: $p_{2} \stackrel{c_{2} ? 1 c_{3} ! 1}{\longrightarrow} p_{2}$ and $p_{2} \stackrel{c_{3} ? 1 c_{1} ? 1}{\longrightarrow} p_{2}$. In a perfect run, there are always as many messages on $c_{1}$ as there are on $c_{2}$ and $c_{3}$ together, and strictly more if a message is lost. Hence a run from $\left\langle p_{1}^{\prime}, p_{2}, \varepsilon, \varepsilon, \varepsilon\right\rangle$ to $\left\langle p_{1}, p_{2}, \varepsilon, \varepsilon, \varepsilon\right\rangle$ must be perfect and witness a solution. Only direct solutions can be accepted since the tally symbols in $c_{3}$ count $\#_{h}$ that cannot be negative.

Reducing $\mathrm{PCP}_{\text {dir }}$ to $T_{4}^{u}$-networks. For $T_{4}^{u}$, we further adapt the idea, again with the count $\#_{h}$ stored on $c_{3}$ but now sent from $P_{2}$ to $P_{1}$. The loops in $P_{1}$ now are

$$
p_{1} \stackrel{c_{1} ! x_{i} c_{2} ! y_{i} 1^{\left|x_{i}\right|}}{\longrightarrow} q_{i} \stackrel{c_{3} ? 1^{\left|y_{i}\right|}}{\longrightarrow} p_{1}
$$

The 1 's on $c_{2}$ are sent back via $c_{3}$ to be matched later by $P_{1}$, thanks to a loop $p_{2} \stackrel{c_{2} ? 1 c_{3} ! 1}{\longrightarrow} p_{2}$. Again a message loss will leave strictly more messages in $c_{1}$ than in $c_{2}$ and $c_{3}$ together, and cannot be recovered from. Only direct solutions can be accepted since the tally symbols in $c_{3}$ count $\#_{h}$.

\section{Fusion for essential channels}

Sections 4 and 5 develop techniques for "simplifying" topologies while preserving the decidability status of reachability problems. We start with a reduction called "fusion".

Let $T=\langle N, R, L, s, d\rangle$ be a network topology. For any channel $c \in C, T-c$ denotes the topology obtained from $T$ by deleting $c$. For any two distinct nodes $P_{1}, P_{2} \in N$, $T\left[P_{1}=P_{2}\right]$ denotes the topology obtained from $T$ by merging $P_{1}$ and $P_{2}$ in the obvious way: channel extremities are redirected accordingly.

Clearly, any MCS with topology $T-c$ can be seen as having topology $T$. Thus $T-c$ has decidable reachability when $T$ has, but the converse is not true in general.

Similarly, any MCS having topology $T$ can be transformed into an equivalent MCS having topology $T\left[P_{1}=P_{2}\right]$ (using the asynchronous product of two control automata). Thus $T$ has decidable reachability when $T\left[P_{1}=P_{2}\right]$ has, but the converse is not true in general. 
For any channel $c$ such that $s(c) \neq d(c)$, we let $T / c$ denote $T[s(c)=d(c)]-c$ and say that $T / c$ is "obtained from $T$ by contracting $c$ ". Hence $T / c$ is obtained by merging $c$ 's source and destination, and then removing $c$.

Since $T / c$ is obtained via a combination of merging and channel removal, there is, in general, no connection between the decidability of reachability for $T$ and for $T / c$. However, there is a strong connection for so-called "essential" channels, as stated in Theorem 4.5 below.

Before we can get to that point, we need to explain what are essential channels and how they can be used.

\subsection{Essential channels are existentially 1-bounded}

In this section, we assume a given MCS $S=\left\langle T, M, Q_{1}, \Delta_{1}, \ldots\right\rangle$ with $T=\langle N, R, L, s, d\rangle$.

Definition 4.1. A channel $c \in C$ is essential if $s(c) \neq d(c)$ and all directed paths from $s(c)$ to $d(c)$ in $T$ go through $c$.

In other words, removing $c$ modifies the connectivity of the directed graph underlying $T$.

The crucial feature of an essential channel $c$ is that causality between the actions of $s(c)$ and the actions of $d(c)$ is constrained. As a consequence, it is always possible to reorder the actions in a run so that reading from $c$ occurs immediately after the corresponding writing to $c$. As a consequence, bounding the number of messages that can be stored in $c$ does not really restrict the system behaviour.

Formally, for $b \in \mathbb{N}$, we say a channel $c$ is $b$-bounded along a run $\pi=\sigma_{0} \stackrel{\delta_{1}}{\rightarrow} \ldots \stackrel{\delta_{n}}{\rightarrow} \sigma_{n}$ if $\left|\sigma_{i}(c)\right| \leq b$ for $i=0, \ldots, n$. We say $c$ is synchronous in $\pi$ if it is 1 -bounded and at least one of $\sigma_{i}(c)$ and $\sigma_{i+1}(c)$ is $\varepsilon$ for all $0 \leq i<n$. Hence a synchronous channel only stores at most one message at a time, and the message is read immediately after it has been written to $c$.

Proposition 4.2. If $c$ is essential and $\pi=\sigma_{0} \stackrel{\delta_{1}}{\rightarrow} \stackrel{\delta_{n}}{\rightarrow} \sigma_{n}$ is a run with $\sigma_{0}(c)=\sigma_{n}(c)=\varepsilon$, then $S$ has a run $\pi^{\prime}$ from $\sigma_{0}$ to $\sigma_{n}$ in which $c$ is synchronous.

This notion is similar to the existentially-bounded systems of [LM04] but is applies to a single channel, not to the whole system.

We prove Proposition 4.2 using techniques and concepts from true concurrency theory and message flow graphs (see, e.g., [HMK $\left.\left.{ }^{+} 05\right]\right)$. With a run $\pi=\sigma_{0} \stackrel{\delta_{1}}{\rightarrow} \ldots \stackrel{\delta_{n}}{\rightarrow} \sigma_{n}$ as above, we associate a set $E=\{1, \ldots, n\}$ of $n$ events, that can be thought of the actions performed by the $n$ steps of $\pi$ : firing a transition and reading or writing or losing a message. Observe that different occurrences of a same transition with same effect are two different events. We simply identify the events with indexes from 1 to $n$. We write $e, e^{\prime}, \ldots$ to denote events, and also use the letters $r$ and $w$ for reading and writing events.

Any $e \in E$ is an event of some process $N(e) \in N$ and we write $E=\bigcup_{P \in N} E_{P}$ the corresponding partition. There exist several (standard) causality relations between events. For every process $P \in N$, the events of $P$ are linearly ordered by $<_{P}: i<_{P} j$ iff $i, j \in E_{P}$ and $i<j$. For every channel $c \in C$, the events that write to or read from $c$ are related by $<_{c}$ with $i<_{c} j$ iff $i$ is an event that writes some $m$ to $c$, and $j$ is the event that reads that 
(occurrence of) $m$. (Here, events that lose messages are considered as internal actions where no channel is involved.) We let $\prec$ (and $\preccurlyeq$ ) denote the transitive (resp. reflexivetransitive) closure of $\bigcup_{P \in N}<_{P} \cup \bigcup_{c \in C}<_{c}$. $(E, \preccurlyeq)$ is then a poset, and $\preccurlyeq$ is called the visual order (also causality order, or dependency order) in the literature. For $e \in E$, we let $\downarrow e$ denote the past of $e$, i.e., the set $\left\{e^{\prime} \in E \mid e^{\prime} \preccurlyeq e\right\}$.

It is well-known that any linear extension $e_{1}, \ldots, e_{n}$ of $(E, \preccurlyeq)$ is causally consistent and can be transformed into a run $\pi^{\prime}=\sigma_{0} \stackrel{e_{1}}{\stackrel{e_{2}}{\longrightarrow}} \cdots$ starting from $\sigma_{0}$. This run ends in $\sigma_{n}$ like $\pi$, though it may go through different intermediary configurations. All the runs obtained by considering different linear extensions are causally equivalent to $\pi$, denoted $\pi \approx \pi^{\prime}$, and they all give rise to the same poset $(E, \preccurlyeq)$.

We now state properties enjoyed by $(E, \preccurlyeq)$ in our context that are useful for proving Proposition 4.2. First, observe that, since the channels are fifo, and since only one process, namely $d(c)$ (resp. $s(c)$ ), is allowed to read from (resp. write to) a channel $c$ :

$$
\left(w_{1}<_{c} r_{1} \text { and } w_{2}<_{c} r_{2}\right) \text { imply }\left(w_{1}<_{s(c)} w_{2} \text { iff } r_{1}<_{d(c)} r_{2}\right) \text {. }
$$

$(\dagger)$ is sometimes taken as a definition of fifo communication.

Another important observation is the following: assume $e \preccurlyeq e^{\prime}$. Then, and since $\preccurlyeq$ is defined as a reflexive-transitive closure, there must be a chain of the form

$$
\theta: e=e_{0} \leq_{P_{0}} e_{0}^{\prime}<_{c_{1}} e_{1} \leq_{P_{1}} e_{1}^{\prime}<_{c_{2}} \ldots<_{c_{l}} e_{l} \leq_{P_{l}} e_{l}^{\prime}=e^{\prime}
$$

where, for $1 \leq i \leq l, s\left(c_{i}\right)=P_{i-1}$ and $d\left(c_{i}\right)=P_{i}$. Hence $T$ has a path $c_{1}, \ldots, c_{l}$ going from $P_{0}$ to $P_{l}$.

Lemma 4.3. If $e_{1} \prec e_{2} \prec e_{3}$ and $c$ is essential, then $e_{1} \nless_{c} e_{3}$.

Proof. By contradiction. Assume $e_{1} \prec e_{2} \prec e_{3}$ and $e_{1}<_{c} e_{3}$ for an essential $c$. Since all paths from $P=N\left(e_{1}\right)=s(c)$ to $P^{\prime}=N\left(e_{3}\right)=d(c)$ go through $c$ (by essentiality), there must exist a pair $w, r \in E$ with $e_{1} \preccurlyeq w<_{c} r \preccurlyeq e_{2}$ or, symmetrically, $e_{2} \preccurlyeq w<_{c} r \preccurlyeq e_{3}$, depending on whether the $w<_{c} r$ pair occurs before or after $e_{2}$ in the chain from $e_{1}$ to $e_{2}$ to $e_{3}$. If $e_{1} \preccurlyeq w<_{c} r \preccurlyeq e_{2} \prec e_{3}$, then $r<_{P^{\prime}} e_{3}$, hence $w<_{P} e_{1}$ using (†). If $e_{1} \prec$ $e_{2} \preccurlyeq w<_{c} r \preccurlyeq e_{3}$, then $e_{1}<P w$, hence $e_{3}<_{P^{\prime}} r$ using ( $\dagger$ ). In both cases we obtain a contradiction.

We now assume that $c$ is essential and that $\pi$ has $\sigma_{0}(c)=\sigma_{n}(c)=\varepsilon$ (hence $E$ has the same number, say $m$, of events reading from $c$ and writing to it). Write $P$ for $s(c)$ and $P^{\prime}$ for $d(c)$. Let $w_{1}<_{P} w_{2} \ldots<_{P} w_{m}$ be the $m$ events that write to $c$, listed in causal order. Let $r_{1}<{ }_{P^{\prime}} e_{2} \ldots<_{P^{\prime}} r_{m}$ be the $m$ events that read from $c$ listed in causal order.

Lemma 4.4. There exists a linear extension of $(E, \preccurlyeq)$ where, for $i=1, \ldots, m, w_{i}$ occurs just before $r_{i}$.

Proof. The linear extension is constructed incrementally. Formally, for $i=1, \ldots, m$, let $E_{i} \stackrel{\text { def }}{=} r_{i}$ and $F_{i} \stackrel{\text { def }}{=} E_{i} \backslash\left\{w_{i}, r_{i}\right\}$. Observe that $F_{1} \subsetneq E_{1} \subseteq F_{2} \cdots F_{i} \subsetneq E_{i} \subseteq F_{i+1}$, with the convention that $F_{m+1}=E$. Every $E_{i}$ is a $\preccurlyeq$-closed subset of $E$, also called a down-cut of $(E, \preccurlyeq)$. Furthermore, $F_{i}$ is a down-cut of $E_{i}$ by Lemma 4.3. Hence a linear extension 
of $F_{i}$ followed by $w_{i} \cdot r_{i}$ gives a linear extension of $E_{i}$, and following it with a linear extension of $F_{i+1} \backslash E_{i}$ gives a linear extension of $F_{i+1}$. Any linear extension of $F_{i+1} \backslash E_{i}$ can be chosen since this subset does not contain reads from, or writes to, $c$.

The linear extension we just built gives rise to a run $\pi^{\prime}$ in which $c$ is synchronous. This concludes the proof of Proposition 4.2.

Observe that when several channels are essential in $T$, it is in general not possible to replace a run $\pi$ with an equivalent $\pi^{\prime}$ where all essential channels are simultaneously synchronous.

\subsection{Decidability by fusion}

We call "fusion" the transformation of $T$ to $T / c$ where $c$ is essential, and "reliable fusion" the special case where $c$ is also a reliable channel.

Theorem 4.5 (Decidability by fusion). Let $c$ be an essential channel in $T$ :

1. $T$ has decidable reachability if $T / c$ has.

2. If $c$ is a reliable channel, then $T / c$ has decidable reachability if $T$ has.

Proof. 1. Let $S$ be a $T$-MCS. We replace it by a system $S^{\prime}$ where $c$ has been removed and where the processes at nodes $P_{1}=s(c)$ and $P_{2}=d(c)$ have been replaced by a larger process that simulate both $P_{1}$ and $P_{2}$ and where communication along $c$ is replaced by synchronizing the sends in $P_{1}$ with the reads in $P_{2}$ (message losses are simulated even more simply by the $P_{1}$ part). $S^{\prime}$ has topology $T / c$ and simulates $S$ restricted to runs where $c$ is synchronous. By Proposition 4.2, this is sufficient to reach any reachable configuration. Since reachability in $S^{\prime}$ is decidable, we conclude that reachability in $S$ is decidable.

2. We now also assume that $c$ is reliable and consider a $(T / c)$-MCS $S$. With $S$ we associate a $T$-MCS $S^{\prime}$ that simulates $S . S^{\prime}$ has two nodes $P_{1}$ and $P_{2}$ where $S$ only had a merged $P$ node.

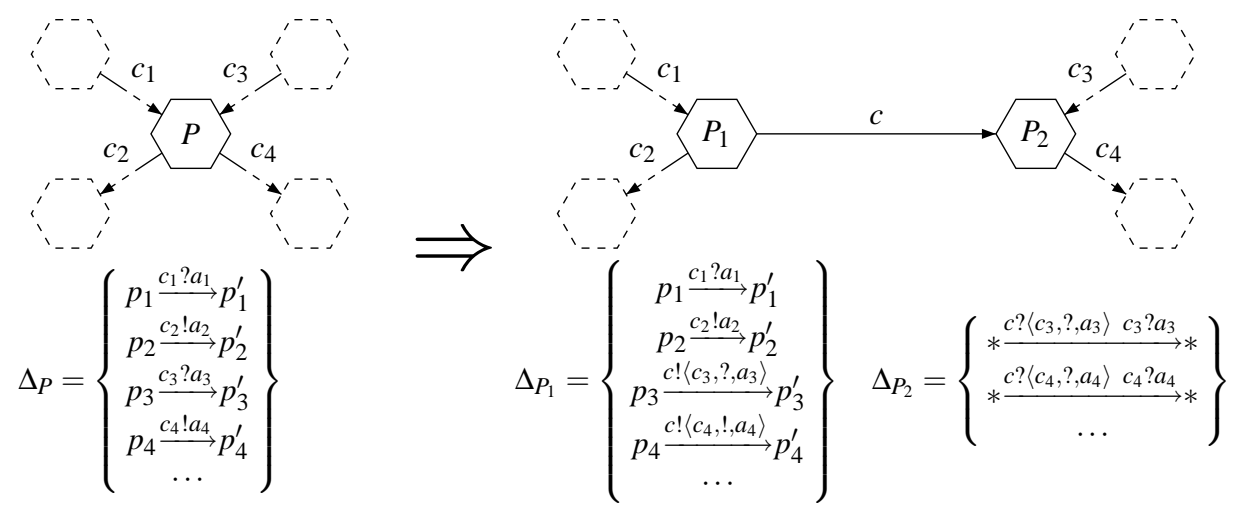

Fig. 3. Associating a $T$-MCS with a $T / c$-MCS 
The construction is illustrated in Fig. 3. Informally, $P_{1}$ inherits states from $P$ and all rules that read from channels $c_{1}$ with $d\left(c_{1}\right)=P_{1}$ in $T$, or write to channels $c_{2}$ with $s\left(c_{2}\right)=P_{1}$. Regarding the other rules, the communication action (reading from some $c_{3}$ or writing to some $c_{4}$ ) is sent to $P_{2}$ via $c . S^{\prime}$ uses an extended alphabet $M^{\prime}$ that extends the message alphabet $M$ from $S$ via $M^{\prime} \stackrel{\text { def }}{=} M \cup(C \times\{?, !\} \times M) . P_{2}$ only has simple loops around a central state $*$ that read communication instructions from $P_{1}$ via $c$ and carry them out.

$S^{\prime}$ simulates $S$ in a strong way. Any step in $S$ can be simulated in $S^{\prime}$, perhaps by two consecutive steps if a communication operation has to transit from $P_{1}$ to $P_{2}$ via $c$. In the other direction, there are some runs in $S^{\prime}$ that cannot be simulated directly by $S$, e.g., when $P_{2}$ does not carry out the instructions sent by $P_{1}$ (or carries them out with a delay). But all runs in $S^{\prime}$ in which $c$ is synchronous are simulated by $S$.

Since runs in which $c$ is synchronous are sufficient to reach any configuration reachable in $S^{\prime}$ (Proposition 4.2), the two-way simulation reduces reachability in $S$ to reachability in $S^{\prime}$, which is decidable if $T$ has decidable reachability.

The usefulness of Theorem 4.5 is illustrated by the following two corollaries.

Corollary 4.6. $T_{1}^{\text {ring }}$ and $T_{3}^{\text {ring }}$ (from Section 2.1) have decidable reachability. $T_{2}^{\text {ring }}$ does not.

Proof. Building $T_{1}^{\text {ring }} / c_{3} / c_{4} / c_{5} / c_{6} / c_{1}$ only fuses essential channels and ends up with a decidable topology (only lossy channels).

Starting with $T_{2}^{\text {ring }}$, we can build $T=T_{2}^{\text {ring }} / c_{3} / c_{4} / c_{5} / c_{6}$ but have to stop there $\left(c_{1}\right.$ is not essential). The resulting $T$, isomorphic to $T_{4}^{u}$ from Fig. 2, does not have decidable reachability. Hence $T_{2}^{\text {ring }}$ does not have decidable reachability since we fused reliable channels only.

With $T_{3}^{\text {ring }}$, it is better to build $T_{3}^{\text {ring }} / c_{3} / c_{4} / c_{6} / c_{1}$. Here too we cannot fuse any more because of $c_{2}^{\prime}$, but the end result is a topology with decidable reachability since $c_{5}$ is lossy. Hence $T_{3}^{\text {ring }}$ has decidable reachability.

Corollary 4.7. A topology in the form of an undirected forest has decidable reachability.

Proof (Sketch). If $T$ is a forest, every channel $c$ is essential, and every $T / c$ is still a forest. Hence $T$ reduces to a topology with lossy channels only.

\section{Splitting along lossy channels}

Let $T_{1}=\left\langle N_{1}, R_{1}, L_{1}, s_{1}, d_{1}\right\rangle$ and $T_{2}=\left\langle N_{2}, R_{2}, L_{2}, s_{2}, d_{2}\right\rangle$ be two disjoint topologies. We say that $T=$ $\langle N, R, L, s, d\rangle$ is a (lossy) gluing of $T_{1}$ on $T_{2}$ if $T$ is a juxtaposition of $T_{1}$ and $T_{2}$ (hence $N=N_{1} \cup N_{2}$ ) with an additional set $L_{3}$ of lossy

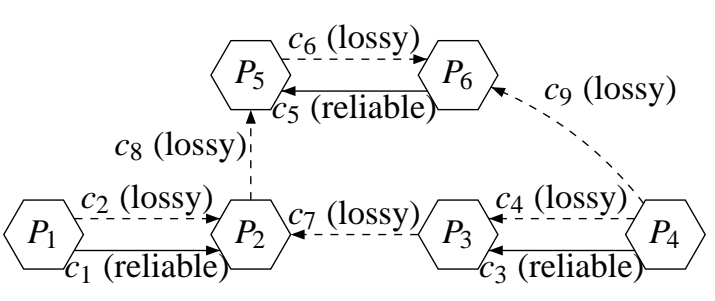

Fig. 4. A topology that splits in three 
channels (hence $R=R_{1} \cup R_{2}$ and $L=L_{1} \cup L_{2} \cup L_{3}$ ) connecting from $T_{1}$ to $T_{2}$ in a unidirectional way: $s\left(L_{3}\right) \subseteq N_{1}$ and $d\left(L_{3}\right) \subseteq N_{2}$.

This situation is written informally " $T=T_{1} \triangleright T_{2}$ ", omitting details on $L_{3}$ and its connections. In practice this notion is used to split a large $T$ into subparts rather than build larger topologies out of $T_{1}$ and $T_{2}$.

Theorem 5.1 (Decidability by splitting). Reachability is decidable for $T_{1} \triangleright T 2$ if, and only if, it is for both $T_{1}$ and $T_{2}$.

The proof of Theorem 5.1 (see Appendix A) uses techniques that are standard for LCS's but that have to be adapted to the more general setting of MCS's.

We can apply Theorem 5.1 to prove that the topology in Fig. 4 has decidable reachability. Indeed, this topology can be split along lossy channels (first $\left\{c_{8}, c_{9}\right\}$, then $c_{7}$ ), giving rise to two copies of $T_{2}^{d}$ (from Fig. 1) and a two-node ring that can be reduced to $T_{1}^{d}$ by fusion.

\section{A complete classification}

In this section, we prove that the results from the previous sections provide a complete classification.

Theorem 6.1 (Completeness). A network topology $T$ has decidable reachability if, and only if, it can be reduced to $T_{2}^{d}$ (from Fig. 1) and LCS's using fusion and splitting only. ${ }^{2}$

Note that, via splitting, the reduction above usually transforms $T$ into several topologies. All of them must be $T_{2}^{d}$ or LCS's for $T$ to have decidable reachability.

The " $\Leftarrow$ " direction is immediate in view of Theorems 4.5.1 and 5.1,

For the " $\Rightarrow$ " direction, we can assume w.l.o.g. that $T$ is reduced, i.e., it cannot be split as some $T_{1} \triangleright T_{2}$, and it does not contain any reliable essential channel (that could be fused).

We now assume, by way of contradiction, that $T$ cannot be transformed, via general fusions, to $T_{2}^{d}$ or to a LCS. From this we show that reachability is not decidable for $T$. When showing this, we sometimes mention three additional transformations ("simplification", "doubling of loops" and "non-essential fusion") that are described in Appendix B. We now start an involved case analysis.

1. Since $T$ cannot be transformed to a LCS, it contains a reliable channel $c_{r}$, linking node $A=s\left(c_{r}\right)$ to node $B=d\left(c_{r}\right)$. We can assume $A \neq B$, otherwise $T$ contains $T_{1}^{u}$ (from Fig. 2) and we conclude immediately with undecidability.

2. $T$ must contain a path $\theta$ of the form $A=P_{0}, c_{1}, P_{1}, c_{2}, \ldots, c_{n}, P_{n}=B$ that links $A$ to $B$ without using $c_{r}$, otherwise $c_{r}$ would be essential, contradicting the assumption that $T$ is reduced. We pick the shortest such $\theta$ (it is a simple path) and we call $T^{\prime}$ the subgraph of $T$ that only contains $\theta, c_{r}$, and the nodes to which they connect.

\footnotetext{
${ }^{2}$ As is well-known, it is possible to further reduce any LCS into $T_{1}^{d}$. However, we preferred a statement for Theorem 6.1 where only our two main transformations are involved.
} 
3. If all $c_{i}$ 's along $\theta$ are reliable, $T^{\prime}$ can be transformed to $T_{2}^{u}$ (from Fig. 2) by reliable fusions, hence $T^{\prime}$, and then $T$ itself, have undecidable reachability. Therefore we can assume that at least one $c_{i}$ along $\theta$ is lossy.

4. Assume that there exist two nodes $P_{i}, P_{j}$ along $\theta$ that are connected via a third path $\theta^{\prime}$ disjoint from $c_{r}$ and $\theta$. We put no restrictions on the relative positions of $P_{i}$ and $P_{j}$ but we assume that $\theta^{\prime}$ is not a trivial empty path if $i=j$. In that case, let $T^{\prime \prime}$ be the subgraph of $T$ that contains $c_{r}, \theta$, and $\theta^{\prime}$, and where all channels except $c_{r}$ are downgraded to lossy if they were reliable. Using simplification and doubling of lossy loops, $T^{\prime \prime}$ can be transformed to an undecidable topology among $\left\{T_{3}^{u}, T_{4}^{u}, T_{5}^{u}, T_{6}^{u}\right\}$. Hence $T^{\prime \prime}$ does not have decidable reachability. Neither has $T$ since taking subgraphs and downgrading channels can only improve decidability.

5. If we are not in case 4 , the nodes along $\theta$ do not admit a third path like $\theta^{\prime}$. Therefore all channels along $\theta$ must be lossy, since we assumed $T$ is reduced. Thus $T^{\prime}$ can be transformed to $T_{2}^{d}$ by general fusion. Since we assumed $T$ cannot be transformed to $T_{2}^{d}, T$ must contain extra nodes or channels beyond those of $T^{\prime}$. In particular, this must include extra nodes since we just assumed that $T$ has no third path $\theta^{\prime}$ between the $T^{\prime}$ nodes. Furthermore these extra nodes must be connected to the $T^{\prime}$ part otherwise splitting $T$ would be possible. There are now several cases.

6. We first consider the case of an extra node $C$ with a reliable channel $c$ from $C$ to $T^{\prime}$. Since $T$ is reduced, $c$ is not essential and there must be a second path $\theta^{\prime}$ from $C$ to $T^{\prime}$. Call $T^{\prime \prime}$ the subgraph of $T$ that only contains $T^{\prime}, C, c$ and $\theta^{\prime}$. Applying non-essential fusion on $c, \theta^{\prime}$ becomes a path between some $P_{i}, P_{j}$ and we are back to case 4 . Hence undecidability.

7. Next is the case of an extra node $C$ with a reliable channel $c$ from $T^{\prime}$ to $C$. Again, since $c$ is not essential, there must be a second path $\theta^{\prime}$ from $T^{\prime}$ to $C$. Again, the induced subgraph $T^{\prime \prime}$ can be shown undecidable as in case 6 , reducing to case 4 .

8. If there is no extra node linked to $T^{\prime}$ via a reliable $c$, the extra nodes must be linked to $T^{\prime}$ via lossy channels. Now the connection must go both ways, otherwise splitting would be possible. The simplest case is an extra node $C$ with a lossy $c$ from $C$ to $T^{\prime}$ and a lossy $c^{\prime}$ from $T^{\prime}$ to $C$. But this would have been covered in case 4 .

9. Finally there must be at least two extra nodes $C$ and $C^{\prime}$, with a lossy channel $c$ from $C$ to $T^{\prime}$ and a lossy $c^{\prime}$ from $T^{\prime}$ to $C^{\prime}$. We can assume that all paths between $T^{\prime}$ and $C, C^{\prime}$ go through $c$ and $c^{\prime}$, otherwise we would be in one of the cases we already considered. Furthermore $C$ and $C^{\prime}$ must be connected otherwise $T$ could be split. There are several possibilities here.

10. If there is a path from $C^{\prime}$ to $C$ we are back to case 4 . Hence undecidability.

11. Thus all paths connecting $C$ and $C^{\prime}$ go from $C$ to $C^{\prime}$. If one such path is made of reliable channels only, reliable fusion can be applied on the induced subgraph, merging $C$ and $C^{\prime}$ and leading to case 8 where undecidability has been shown. If they all contain one lossy channel, $T$ can be split, contradicting our assumption. that it is reduced.

We have now covered all possibilities when $T$ is reduced but cannot be transformed to a LCS or to $T_{2}^{d}$. In all cases is has been shown that reachability is not decidable for $T$. This concludes the proof of Theorem 6.1. 


\section{A classification algorithm}

Theorem 7.1 (Polynomial-time classification). There exists a polynomial-time algorithm that classifies topologies according to whether they have decidable reachability.

The algorithm relies on Theorem 6.1:

Stage 1: Starting from a topology $T$, apply splitting and reliable fusion as much as possible. When several transformations are possible, pick any of them nondeterministically. At any step, the transformation reduces the size of the topologies at hand, hence termination is guaranteed in a linear number of steps. At this stage we preserved decidability in both directions, hence $T$ has decidability iff all the reduced topologies $T_{1}, \ldots, T_{n}$ have.

Stage 2: Each $T_{i}$ is now simplified using general fusion (not just reliable fusion). If this ends with a LCS or with $T_{2}^{d}$, decidability for $T_{i}$ has been proved. When fusion can be applied in several ways, we pick one nondeterministically: a consequence of Theorem 6.1's proof is that these choices lead to the same conclusion when starting from a system that cannot be reduced with splitting or reliable fusion. Thus stage 2 terminates in a linear number of steps. When it terminates, either every $T_{i}$ has been transformed into a LCS or $T_{2}^{d}$, and we conclude that reachability is decidable for $T$, or one $T_{i}$ remains unsimplified and we conclude that reachability is not decidable for $T$.

We observe that when stage 1 finishes, there will never be any new opportunity for reliable fusion or for splitting since stage 2, i.e., general fusion, does not create or destroy any path between nodes.

\section{Concluding remarks}

Summary. We introduced mixed channel systems, i.e., fifo channel systems where both lossy and reliable channels can be combined in arbitrary topologies. These systems are a generalization of the lossy channel system model (where all channels are lossy and where reachability is decidable) and of the standard model (with unbounded reliable fifo channels, where reachability is undecidable).

For mixed systems, we provide a complete classification of the network topologies according to whether they lead to decidable reachability problems or not. Our main tool are reductions methods that transform a topology into simpler topologies with an equivalent decidability status. These reductions produce small basic topologies for which the decidability status is established in Section 3.

Directions for future work. At the moment our classification is given implicitly, via a simplification procedure. A more satisfactory classification would be a higher-level description, in the form of a structural criterion, preferably expressible in logical form (or via excluded minors, ...). Obtaining such a description is our more pressing objective.

Beyond this issue, the two main avenues for future work are extending the MCS model (e.g., by considering other kinds of unreliability in the style of [CFP96], or by allowing guards in the style of [BBS06], etc.) and considering questions beyond just reachability and safety (e.g., termination and liveness). 


\section{References}

[ABPJ05] P. A. Abdulla, C. Baier, S. Purushothaman Iyer, and B. Jonsson. Simulating perfect channels with probabilistic lossy channels. Information and Computation, 197(12):22-40, 2005.

[ABRS05] P. A. Abdulla, N. Bertrand, A. Rabinovich, and Ph Schnoebelen. Verification of probabilistic systems with faulty communication. Information and Computation, 202(2):141-165, 2005.

[ACBJ04] P. A. Abdulla, A. Collomb-Annichini, A. Bouajjani, and B. Jonsson. Using forward reachability analysis for verification of lossy channel systems. Formal Methods in System Design, 25(1):39-65, 2004.

[AJ96] P. A. Abdulla and B. Jonsson. Verifying programs with unreliable channels. Information and Computation, 127(2):91-101, 1996.

[BBS06] C. Baier, N. Bertrand, and Ph. Schnoebelen. On computing fixpoints in wellstructured regular model checking, with applications to lossy channel systems. In Proc. LPAR 2006, volume 4246 of Lecture Notes in Artificial Intelligence, pages 347-361. Springer, 2006.

[BBS07] C. Baier, N. Bertrand, and Ph. Schnoebelen. Verifying nondeterministic probabilistic channel systems against $\omega$-regular linear-time properties. ACM Trans. Computational Logic, 9(1), 2007.

$\left[\mathrm{BMO}^{+}\right.$08] P. Bouyer, N. Markey, J. Ouaknine, Ph. Schnoebelen, and J. Worrell. On termination for faulty channel machines. In Proc. STACS 2008, pages 121-132, 2008.

[BZ83] D. Brand and P. Zafiropulo. On communicating finite-state machines. Journal of the ACM, 30(2):323-342, 1983.

[CF05] G. Cécé and A. Finkel. Verification of programs with half-duplex communication. Information and Computation, 202(2):166-190, 2005.

[CFP96] G. Cécé, A. Finkel, and S. Purushothaman Iyer. Unreliable channels are easier to verify than perfect channels. Information and Computation, 124(1):20-31, 1996.

[CS07] P. Chambart and $\mathrm{Ph}$. Schnoebelen. Post embedding problem is not primitive recursive, with applications to channel systems. In Proc. FST\&TCS 2007, volume 4855 of Lecture Notes in Computer Science, pages 265-276. Springer, 2007.

[CS08a] P. Chambart and $\mathrm{Ph}$. Schnoebelen. The $\omega$-regular Post embedding problem. In Proc. FOSSACS 2008, volume 4962 of Lecture Notes in Computer Science, pages 97-111. Springer, 2008.

[CS08b] P. Chambart and Ph. Schnoebelen. The ordinal recursive complexity of lossy channel systems. In Proc. LICS 2008. IEEE Comp. Soc. Press, 2008.

[Fin94] A. Finkel. Decidability of the termination problem for completely specificied protocols. Distributed Computing, 7(3):129-135, 1994.

$\left[\mathrm{HMK}^{+} 05\right]$ J. G. Henriksen, M. Mukund, K. N. Kumar, M. A. Sohoni, and P. S. Thiagarajan. A theory of regular MSC languages. Information and Computation, 202(1):1-38, 2005.

[JJ93] T. Jéron and C. Jard. Testing for unboundedness of fifo channels. Theoretical Computer Science, 113(1):93-117, 1993.

[JL07] M. Jurdziński and R. Lazić. Alternation-free modal mu-calculus for data trees. In Proc. LICS 2007, pages 131-140. IEEE Comp. Soc. Press, 2007.

[Kur06] A. Kurucz. Combining modal logics. In P. Blackburn, J. van Benthem, and F. Wolter, editors, Handbook of Modal Logics, volume 3, chapter 15, pages 869-926. Elsevier Science, 2006.

[LM04] M. Lohrey and A. Muscholl. Bounded MSC communication. Information and Computation, 189(2):160-181, 2004. 
[LW08] S. Lasota and I. Walukiewicz. Alternating timed automata. ACM Trans. Computational Logic, 9(2), 2008.

[OW07] J. Ouaknine and J. Worrell. On the decidability and complexity of Metric Temporal Logic over finite words. Logical Methods in Comp. Science, 3(1):1-27, 2007.

[Pac87] J. K. Pachl. Protocol description and analysis based on a state transition model with channel expressions. In Proc. PSTV '87, pages 207-219. North-Holland, 1987.

[Sch02] Ph. Schnoebelen. Verifying lossy channel systems has nonprimitive recursive complexity. Information Processing Letters, 83(5):251-261, 2002.

[Sch04] Ph. Schnoebelen. The verification of probabilistic lossy channel systems. In C. Baier et al., editors, Validation of Stochastic Systems - A Guide to Current Research, volume 2925 of Lecture Notes in Computer Science, pages 445-465. Springer, 2004. 


\section{A Proofs for Section 5}

This section proves Theorem 5.1, i.e., " $T_{1} \triangleright T_{2}$ has decidable reachability iff $T_{1}$ and $T_{2}$ have", where $T_{1} \triangleright T_{2}$ is a juxtaposition of $T_{1}$ and $T_{2}$ with additional glue in the form of lossy channels with source in $T_{1}$ and destination in $T_{2}$.

First observe that the " $\Rightarrow$ " direction is immediate since $T_{1}$ and $T_{2}$ are subgraphs of $T$.

For the " $\Leftarrow$ " direction, we assume $T=T_{1} \triangleright T_{2}$ with $T, T_{1}$ and $T_{2}$ as in Section 5 . We consider a MCS $S$ with topology $T$. From $S$ we extract two subsystems $S_{1}$ and $S_{2}$ with topologies $T_{1}^{\prime}$ and $T_{2}^{\prime}$ that are slight augmentations of $T_{1}$ and $T_{2}$. More precisely, $T_{1}^{\prime}$ is $T_{1}$ augmented with the interface channels $c_{1}, \ldots, c_{k}$ from $L_{3}$, and with dummy extra processes $D_{1}, \ldots, D_{k}$, one for each $c_{i} \in L_{3}$, so that $d\left(c_{i}\right)=D_{i}$ is not left undefined. $T_{2}^{\prime}$ is $T_{2}$ augmented in a similar way, this time with $s\left(c_{i}\right)=D_{i}$. The MCS's $S_{1}$ and $S_{2}$ are the restrictions of $S$ to $T_{1}^{\prime}$ and $T_{2}^{\prime}$ assuming that the extra processes $D_{1}, \ldots, D_{k}$ are inactive.

Observe that, for $i=1,2$, the channels in $L_{3}$ are essential in $T_{i}^{\prime}$ (also note that $T_{i}^{\prime}$ is in general not a subgraph of $T$ since different interface channels in $L_{3}$ may share a common source or a common destination). Since applying fusion on $L_{3}$-channels gives exactly $T_{i}$, and since we assumed reachability is decidable for $T_{i}$, we conclude it is for $T_{i}^{\prime}$ too by Theorem 4.5 .

We now show how to decide reachability for $S$ assuming that reachability is decidable for topologies $T_{1}^{\prime}$ and $T_{2}^{\prime}$, hence for MCS's $S_{1}$ and $S_{2}$.

A configuration $\sigma$ of $S$ can be written under the form $\left\langle\sigma^{1}, \sigma^{2}, u_{1}, \ldots, u_{k}\right\rangle$ where $\sigma^{1}$ is the restriction of $\sigma$ to $T_{1}, \sigma^{2}$ is the restriction to $T_{2}$, and $u_{1}, \ldots, u_{k}$ are the contents of the extra channels from $L_{3}$. (In particular, the contents of channels in $R_{i} \cup L_{i}$ are part of $\left.\sigma^{i}\right)$.

Lemma A.1. Let $\sigma_{\text {init }}=\left\langle\sigma_{\text {init }}^{1}, \sigma_{\text {init }}^{2}, \varepsilon, \ldots, \varepsilon\right\rangle$ and $\sigma_{\text {final }}=\left\langle\sigma_{\text {final }}^{1}, \sigma_{\text {final }}^{2}, \varepsilon, \ldots, \varepsilon\right\rangle$ be two configurations of $S$ with empty channels. There is a run $\sigma_{\text {init }} \stackrel{*}{\rightarrow} \sigma_{\text {final }}$ in $S$ if, and only if, there is a tuple $\left\langle u_{1}, \ldots, u_{k}\right\rangle$ such that $S_{1}$ has a run $\left\langle\sigma_{\text {init }}^{1}, \varepsilon, \ldots, \varepsilon\right\rangle{ }^{*} \rightarrow\left\langle\sigma_{\text {final }}^{1}, u_{1}, \ldots, u_{k}\right\rangle$ and $S_{2}$ has a run $\left\langle\sigma_{\text {init }}^{2}, u_{1}, \ldots, u_{k}\right\rangle \stackrel{*}{\rightarrow}\left\langle\sigma_{\text {final }}^{2}, \varepsilon, \ldots, \varepsilon\right\rangle$.

Proof (Sketch). Indeed, since the steps in the $S_{1}$ part of $S$ never depend on the steps in the $S_{2}$ part (interface channels in $L_{3}$ only go from $S_{1}$ to $S_{2}$ ), it is always possible to use all the $S_{1}$ steps first and the $S_{2}$ steps later.

Lemma A.2. The following problems are decidable:

(1) Given some $\left\langle u_{1}, \ldots, u_{k}\right\rangle \in\left(M^{*}\right)^{k}$, does $S_{1}$ have a run $\left\langle\sigma_{\text {init }}^{1}, \varepsilon, \ldots, \varepsilon\right\rangle \stackrel{*}{\rightarrow}\left\langle\sigma_{\text {final }}^{1}, u_{1}, \ldots, u_{k}\right\rangle$ ?

(2) Given some $\left\langle u_{1}, \ldots, u_{k}\right\rangle \in\left(M^{*}\right)^{k}$, does $S_{2}$ have a run $\left\langle\sigma_{\text {init }}^{2}, u_{1}, \ldots, u_{k}\right\rangle \stackrel{*}{\rightarrow}\left\langle\sigma_{\text {final }}^{2}, \varepsilon, \ldots, \varepsilon\right\rangle$ ?

(3) Given some regular languages $R_{1}, \ldots, R_{k} \subseteq M^{*}$, does there exists a tuple $\left\langle u_{1}, \ldots, u_{k}\right\rangle \in$ $R_{1} \times \cdots \times R_{k}$ such that $S_{2}$ has a run $\left\langle\sigma_{\text {init }}^{2}, u_{1}, \ldots, u_{k}\right\rangle \stackrel{*}{\rightarrow}\left\langle\sigma_{\text {final }}^{2}, \varepsilon, \ldots, \varepsilon\right\rangle$ ?

Proof. (1) is almost immediate since reachability is decidable in $T_{1}^{\prime}$. Since we insist on asking reachability questions with empty channels in the initial and final configurations, we have to program the extra components $D_{1}, \ldots, D_{k}$ so that they empty the $c_{i}$ and check that they contained $u_{i}$ and only accept if this is the case. The resulting system is still a $T_{1}^{\prime}$ system. 
For (2), the same idea applies but this time the $D_{i}$ 's fill the interface channels with the $u_{i}$. Ensuring that $u_{i}$ is really inserted in $c_{i}$ is done by upgrading the interface channels from lossy to reliable channels. This does not impact the decidability of reachability since it is established by fusing essential channels and reducing to $T_{2}$.

For (3) we program the $D_{i}$ 's so that they nondeterministically write one $u_{i} \in R_{i}$ in $c_{i}$. Since $R_{i}$ is regular, a finite-state $D_{i}$ can do the generation. Hence we reduced (3) to a reachability question on a decidable topology ( $T_{2}^{\prime}$ with reliable interface channels).

Lemma A.3. The set $R \subseteq\left(M^{*}\right)^{k}$ of all minimal (w.r.t. the subword ordering) tuples $\left\langle u_{1}, \ldots, u_{k}\right\rangle$ allowing $\left\langle\sigma_{\text {init }}^{2}, u_{1}, \ldots, u_{k}\right\rangle \stackrel{*}{\rightarrow}\left\langle\sigma_{\text {final }}^{2}, \varepsilon, \ldots, \varepsilon\right\rangle$ is finite and can be computed effectively.

Proof. $R$ is finite since the subword ordering is a well-quasi-order (Higman's Lemma).

Regarding its computation, we cannot apply the backward reachability algorithm for LCS's since $T_{2}^{\prime}$ may contain reliable channels. However, by Lemma A.2.(2), we can check any candidate tuple. Therefore it is possible to build $R$ incrementally by enumerating all candidate tuples. Enumerating them in order of increasing length ensures that only minimal tuples are retained.

This procedure is bound to eventually build $R$ (since it is finite) and there only remains to ensure termination by detecting when the current $R$ is complete. This can be done using Lemma A.2.(3): the set $R^{\prime}$ of all tuples that do not contain a tuple from $R$ as subword is a regular language, being the complement of the upward-closure of a finite set. Thus we can decide whether $R^{\prime}$ contains some tuple that is not yet accounted for in $R$. One detail is that $R^{\prime}$, though regular, is not in general a product $R_{1}^{\prime} \times \cdots \times R_{k}^{\prime}$ of regular languages, one for each part of the tuple. However it is well-known that such sets are a finite union $\sum_{i} R_{1, i}^{\prime} \times \cdots \times R_{k, i}^{\prime}$ of products of regular languages.

We now have enough tools to implement Lemma A.1 and thereby decide reachability for $S$. We compute $R$ and check, using Lemma A.2.1, that one of the tuples in $R$ is reachable with $S_{1}$. Observe that restricting to minimal tuples does not invalidate the algorithm: $c_{1}, \ldots, c_{k}$ being lossy, the set of tuples that $S_{1}$ can write there is downwardclosed.

\section{B Some additional transformations}

This section describes additional transformations and how they preserve decidability of reachability. The correctness proofs are only sketched in this extended abstract, but the missing parts are easy to fill in since the transformations are similar to existing ones.

We list these transformations for the sake of completeness (they are used in the proof of Theorem 6.1) but the reader should understand that they do not occur in the classification algorithm, or in the statement of the classification theorem, where only essential fusion and splitting are needed.

1. Double lossy loops. We say that $T$ has a double lossy loop if there are distinct $c, c^{\prime} \in$ $L$ with $s(c)=d(c)=s\left(c^{\prime}\right)=d\left(c^{\prime}\right)$. 
Lemma B.1. If $c$ and $c^{\prime}$ are a double lossy loop in $T$ then reachability is decidable for $T$ if, and only if, it is for $T-c^{\prime}$.

Proof (Idea). A single loop can simulate two loops the way a single lossy loop can simulate an arbitrary LCS: we concatenate the contents of the two original channels in the remaining one, using special markers to separate the two contents (see, e.g., [Sch02, Section 5]). Acting on one part of the contents requires rotating the contents of the channels, and this can be achieved with the help of the markers. The markers are inserted at the start of the run, and removed at the end. If they are lost during the simulation, correct simulation cannot be guaranteed, but it will be impossible to reach an accepting state. Hence the simulation is correct for reachability questions. The new observation is that it remains correct with an arbitrary mixed topology around the two loops under consideration.

Remark B.2. Paradoxically, we do not use Lemma B.1 for simplifying systems. Rather we use it for doubling loops, which may prove useful when we try to obtain basic topologies from Fig. 2 via simplification (see below). Hence it is important that Lemma B.1 preserves decidability in both directions.

2. Simplification. Let $T$ be a topology with a lossy channel system $c$ between two nodes $P_{1}$ and $P_{2}$. The simplification of $T$ by $c$ is a topology $T^{\prime}$ where $c$ has been removed and where all channels $c^{\prime}$ with $s\left(c^{\prime}\right)=P_{2}$ in $T$ are redirected and have $s\left(c^{\prime}\right)=P_{1}$ in $T^{\prime}$.

Lemma B.3. Reachability is decidable for $T^{\prime}$ if it is for $T$.

Proof (Idea). $T^{\prime}$ misses many features of $T$, which only improves decidability. The features of $T^{\prime}$ that $T$ misses are the channels $c^{\prime}$ from $P_{1}$ to some $P$ that go from $P_{2}$ to $P$ in $T$. In $T$, these can be simulated by a standard multiplexing trick going through $P_{2}$ via $c$.

3. Non-essential fusion. Let $c$ be a reliable channel from $P_{1}$ to $P_{2}\left(P_{1} \neq P_{2}\right)$ in some topology $T$. Assume that there is an additional path from $P_{1}$ to $P_{2}$ that does not use $c$ (hence $c$ is not essential). Further assume that this path only contains lossy channels, and that there is no other path from $P_{1}$ to $P_{2}$.

Lemma B.4. Reachability is decidable for $T / c$ if it is for $T$.

Proving Lemma B.4 is quite different from proving Theorem 4.5. It uses the same simulation we use in [CS07] to link $T_{2}^{d}$ and $T_{1}^{d}$, but this time in a more general context since extra channels and processes may occur in $T$. 